\title{
Effect of Vaccination on Influenza Infection Rate on KSA
}

\author{
Mohammed Ahmed Garout ${ }^{1}$, Imad A AlJahdali ${ }^{1}$, Magda Rmadan Abdultawab ${ }^{2,}$, \\ Rana Mohammed Garout ${ }^{3}$, Raha Mohammed Garout ${ }^{3}$ \\ ${ }^{1}$ Community Medicine \& Public Health, Faculty of Medicine, Umo Alqurah University, Makkah, KSA \\ ${ }^{2}$ Microbiology \& immunology \& Infection Control, Faculty of Medicine, Ainshams University, Cairo, Egypt \\ ${ }^{3}$ Ibn Sina National Collage for Medical Studies, Jeddah, KSA, Saudi Arabia \\ Email address: \\ Ramadan_magda@yahoo.com (M. R. Abdultawab) \\ ${ }^{*}$ Corresponding author
}

\section{To cite this article:}

Mohammed Ahmed Garout, Imad A AlJahdali, Magda Ramadan Abdultawab, Rana Mohammed Garout, Raha Mohammed Garout. Effect of Vaccination on Influenza Infection Rate on KSA. International Journal of Infectious Diseases and Therapy. Vol. 4, No. 2, 2019 , pp. 18-22. doi: $10.11648 /$ j.jijidt.20190402.11

Received: May 16, 2019; Accepted: June 14, 2019; Published: July 11, 2019

\begin{abstract}
Influenza is an acute respiratory disease characterized in its full form by the sudden onset of high fever, coryza, cough, headache, malaise, and inflammation of the upper respiratory tree and trachea. In most cases, pneumonic involvement is not clinically prominent. Aim of study: this Review, we examine the influenza virus infection in cross section study in Riyadh city KSA, This knowledge is used for risk assessments for future, and study effect of influenza vaccine and its implication against influenza virus infection rate STUDY METHODS: A cluster randomized trial involving 1271 children and adolescents and adult, age group between 36 months to 60 years who attend for SMC (Specialized medical center hospital) hospital in ER from January 1 2018, and ended December 31, 2018. History was taken for received vaccine or not, and risk factor diseases (Bronchial Asthma, Diabetics, Heart disease, pneumonia, Immunocompromised). NASOPHARNGEAL swab for: Diagnostic tests: rapid antigen testing, reverse transcription polymerase chain reaction (RT-PCR), and rapid molecular assays, and Follow-up for outcome in infected cases Results: the study revealed that 361of 1271 (28.4\%) had influenza, 89 patient had H1N1 (7\%), 128 patients had influenza A (10.07\%) \& 144 patients had influenza B (11.32\%), one case had H1N1 and Influnza B Detected, $61 \%$ had received vaccination., Among 361 diseased cases 137 receive vaccine (37.9\%), outcome of case did not need hospital admission, complete recovery for a protective effectiveness of $61 \%$ (95\% confidence interval [P $=.03$ ). overall protective effectiveness of influenza vaccine was $59.3 \%$ in children and $66.5 \%$ in adult so over all protection $63 \%$, recommendation Routine annual influenza vaccination is recommended for all persons age 6 months and older.
\end{abstract}

Keywords: Influenza, Vaccination, Protective Effectiveness, Virus Infection, Respiratory

\section{Introduction}

Influenza is an acute respiratory disease characterized in its full form by the sudden onset of high fever, coryza, cough, headache, prostration, malaise, and inflammation of the upper respiratory tree and trachea. In most cases, pneumonic involvement is not clinically prominent. Acute symptoms and fever often persist for 7 to 10 days. Weakness and fatigue may linger for weeks. Influenza usually occurs in winter outbreaks or epidemics (in temperate climates). People of all ages are afflicted, but the prevalence is greatest in school-age children, Croup (laryngotracheitis) can be a serious complication in small children. Influenza A and B viruses are the most common causes of influenza-like illness (ILI), but other pathogens also cause ILI, including influenza $\mathrm{C}$ viruses, parainfluenza viruses, respiratory syncytial viruses, At the peak of an influenza epidemic, approximately one-third of isolates from patients with ILI will be positive for influenza A [1].

Influenza viruses are RNA viruses from the family Orthomyxoviridae, and have a worldwide distribution., Influenza viruses are usually classified into three types: A, B and $\mathrm{C}$, according to differences in antigenic and biologic properties. There is also evidence of a fourth type D [2].

Influenza A viruses are further divided into subtypes. The subtypes are determined by two glycoproteins on the virus 
surface, haemagglutinin (HA) (H1-H18) and neuraminidase (NA) (N1-N11). Antibodies against these glycoproteins are associated with immunity against influenza, Type B viruses cause somewhat less severe disease and tend to cause fewer complications than some type A viruses. Type B does not have subtypes but two antigenically distinct lineages: Victoria and Yamagata. Type A and B viruses are the predominant virus types causing disease in humans, At any one time there is a mix of influenza viruses circulating in the human population. Since the most recent influenza pandemic in 2009 seasonal influenza consists of variable mixes of influenza A (H3N2), A (H1N1) pdm09 - the latter caused the 2009 pandemic. The genome of influenza viruses is subject to a significant spontaneous mutation rate, known as antigenic drift. This results in gradual changes of the human seasonal influenza viruses [3].

In addition, the influenza $\mathrm{A}$ and $\mathrm{B}$ genome consists of eight separate RNA segments. Re-assortment of the genome segments results in considerable antigenic variability, particularly of the HA of the influenza A viruses. Influenza A can also be transmitted interchangeably between humans and swine, and from birds to humans [4]. In birds H1-H16 and N1-N9 have been detected. All these influenza A viruses that have been detected in mammals or poultry originated at some point from wild aquatic birds. H17-18 and N10-11 have been detected in bats, Only type A viruses are able to cause pandemics as they have a reservoir in animals. Pandemics are the result of larger genetic changes called antigenic shift. A shift can occur e.g. through inclusion of HA and NA subtypes from avian or swine origin by re-assortment, i.e. the exchange of at least one RNA segment. Re-assortments are not that rare but only very occasionally lead to a viable influenza A virus, with ability to infect humans, cause disease in humans, cause sustained person-to-person transmission, and for which many or most humans have little or no immunity. That is then a pandemic strain [4].

Influenza Pandemics of the 20th and 21st Century In some cases the disease becomes more severe due to the influenza virus infection or a secondary, usually bacterial, infection e.g. by Streptococcus pneumoniae or Staphylococcus aureus. This most commonly manifests itself in pneumonia, and at times even death. Severe disease may also include myocarditis or encephalitis, which may be fatal. Overall influenza-associated mortality has been estimated to 13.8 per 100,000 person-years [5].

WHO, through the WHO GISRS system, in collaboration with other partners, monitors influenza activity globally, recommends seasonal influenza vaccine compositions twice a year for the Northern and Southern hemisphere influenza seasons, guides countries in tropical and subtropical areas to choose vaccine formulations (Northern hemisphere vs. Southern hemisphere), to support decisions for timing of vaccination campaigns, and to support Member States to develop prevention and control strategies [6].

WHO works to strengthen national, regional and global influenza response capacities including diagnostics, antiviral susceptibility monitoring, disease surveillance and outbreak responses, and to increase vaccine coverage among high risk groups and prepare for the next influenza pandemic. The World Health Organization (WHO) has recommended the composition of the trivalent influenza vaccine (TIV) for the southern hemisphere winter 2018 influenza season as an A/Michigan/45/2015 (H1N1) pdm09 like virus; an A/Singapore/INFIMH-16-0019/2016 (H3N2)-like virus; and, a B/Phuket/3073/2013-like virus Yamagata lineage [7, 8].

The Centers for Disease Control and Prevention (CDC) recommends annual flu vaccination for everyone age 6 months or older [9].

Aim of study:

In this study we examine the influenza virus in cross section study in Riyadh city KSA, This knowledge is used for risk assessments for future, vaccine implication against influenza virus. To assess effectiveness of vaccinating in children and adolescents \&adult for prevention of influenza infection rate.

\section{Study Methods}

\subsection{Design, Setting, and Participants}

A randomized study involving 1298 children and adolescents \& adult, aged between 36 months to 60 years who attend for SMC hospital in ER in period from January 1 2018, and ended December 31, 2018. \&history was taken for received vaccine or did not receive the study vaccine. Follow-up.

\subsection{Main Outcome Measures}

Confirmed influenza A and B infection, H1N1 using a real-time reverse transcriptase polymerase chain reaction (RT-PCR) assay, NASOPHARNGEAL swab for: Diagnostic tests rapid antigen testing, reverse transcription polymerase chain reaction (RT-PCR), and rapid molecular assays. Sensitivity and specificity of any test for influenza might vary by the laboratory that performs the test, Rapid influenza diagnostic tests (RIDTs) provide results within approximately 15 minutes. Most of the rapid influenza diagnostic tests that can be done in a physician's office are approximately 50$70 \%$ sensitive for detecting influenza and approximately greater than $90 \%$ specific. Therefore, false negative results are more common than false positive results, especially during peak influenza activity in the community. Rapid molecular assays can produce results in approximately 20 minutes with high sensitivity and specificity. 3-molecular assays may detect influenza viral RNA in respiratory tract specimens for longer periods after illness onset than antigen detection assays). For hospitalized patients with lower respiratory tract disease and suspected influenza, lower respiratory tract specimens should be collected and tested for influenza viruses by RT-PCR because influenza viral shedding in the lower respiratory tract may be detectable for longer periods than in the upper respiratory tract, RT-PCR Roche Cobas Influenza A/B was cleared and CLIA-waived by FDA for use with nasopharyngeal swabs only. 


\subsection{Molecular Assays}

Reverse Transcription-Polymerase Chain Reaction (RTPCR) and other molecular assays can identify the presence of influenza viral RNA in respiratory specimens with very high sensitivity and specificity. Some molecular assays are able to detect and discriminate between infections with influenza A and $\mathrm{B}$ viruses; other tests can identify specific seasonal influenza A virus subtypes [A (H1N1)]. These assays can yield results in approximately 1-8 hours. Notably, the detection of influenza viral RNA by these assays does not necessarily indicate detection of viable infectious virus or ongoing influenza viral replication.

\section{Results}

The study revealed that 362of 1271 (28.4\%) had influenza, 89 patient had H1N1 (7\%), 128 patients had influenza A (10.07\%) and 144 patients had influenza B (11.32\%), Influenza H1N1 and Influenza B Detected in one case, 61\% of study group had vaccination. Among all study participants (those who were and those who were not vaccinated), --362of $1271(-28.4 \%)$, infection with influenza in the group who were had influenza vaccine (81 out of 199 had influenza infection in children $(40.7 \%)$, and 56 were vaccinated with influenza vaccine out of 162 of diseased with influenza infection in adult (34.5\%), overall protective effectiveness of $59.3 \%$ in children and $66.5 \%$ in adult so over all protection $63 \%(95 \% \mathrm{CI}, \mathrm{P}=.04)$. No serious vaccine adverse events were observed.

Table 1. Total number of case enter in study.

\begin{tabular}{ll}
\hline & Total \# \\
\hline Influenza A (H1N1) is Detected & 89 \\
Influenza A RNA is Detected & 128 \\
Influenza B RNA is Detected & 144 \\
Sample Leakage & 6 \\
Not Detected & 902 \\
Influenza H1N1 and Influenza B Detected & 1 \\
Total swab sample & 1270 \\
\hline
\end{tabular}
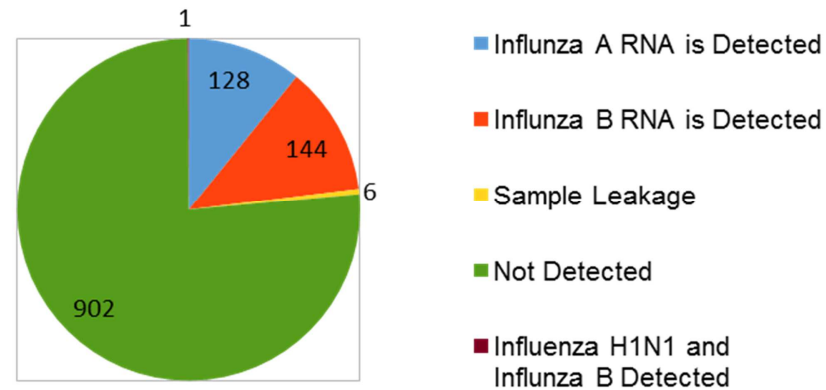

Figure 1. Total number of case enter in study.

Table 2. Effect of Influenza Vaccination of Children (3 monthes -14 years old) on Infection Rates.

\begin{tabular}{|c|c|c|c|c|c|}
\hline Month & Influenza A & Influenza B & H1N1 & VACCINATION & Disease \\
\hline January & 6 & 5 & 1 & 5 & 1 \\
\hline February & 5 & 6 & 2 & 8 & 0 \\
\hline march & 6 & 5 & 1 & 9 & 1 \\
\hline April & 3 & 4 & 2 & 5 & 3 \\
\hline may & 4 & 4 & 2 & 4 & 0 \\
\hline June & 5 & 4 & 3 & 6 & 0 \\
\hline July & 6 & 6 & 2 & 6 & 1 \\
\hline September & 5 & 12 & 1 & 7 & 1 \\
\hline October & 15 & 15 & 5 & 9 & 1 \\
\hline November & 12 & 10 & 4 & 8 & 1 \\
\hline December & 8 & 13 & 5 & 10 & 2 \\
\hline TOTAL & 80 & 90 & 29 & 81 & 11 \\
\hline
\end{tabular}

$\mathrm{P}>0.005$.

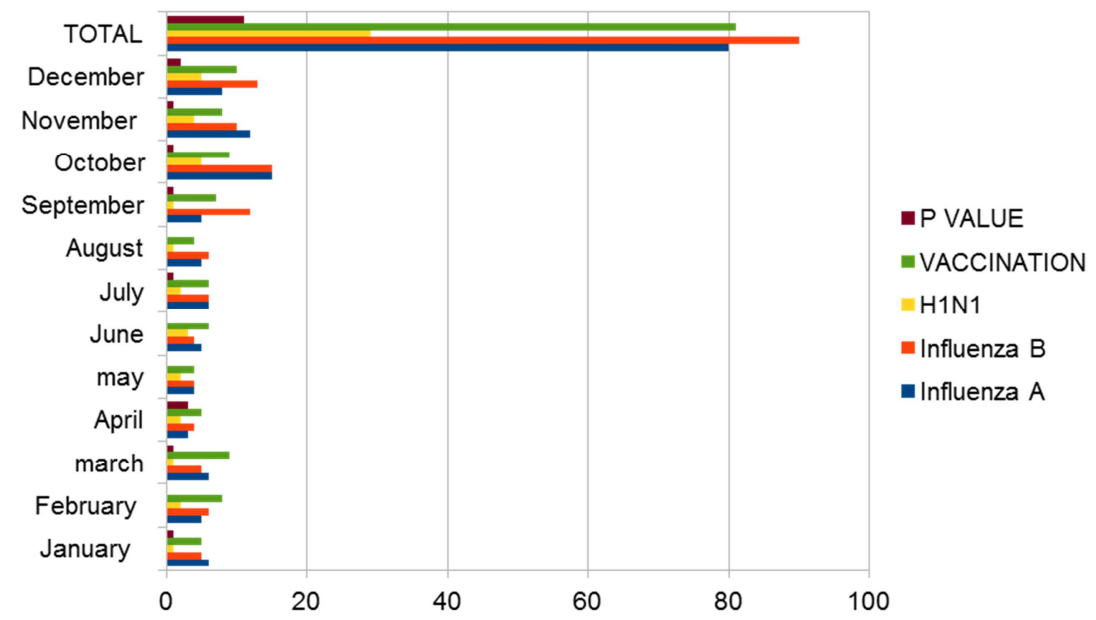

Figure 2. Effect of Influenza Vaccination of Children (3 monthes -14 years old) on Infection Rates. 
Table 3. Effect of Influenza Vaccination of adult on Infection Rates.

\begin{tabular}{|c|c|c|c|c|c|}
\hline Month & Influenza A & Influenza B & H1N1 & VACCINATION & Disease \\
\hline January & 4 & 1 & 2 & 1 & 1 \\
\hline February & 2 & 1 & 1 & 2 & 0 \\
\hline march & 3 & 3 & 4 & 3 & 2 \\
\hline April & 2 & 2 & 2 & 2 & 1 \\
\hline may & 2 & 1 & 3 & 2 & 0 \\
\hline June & 3 & 3 & 1 & 1 & 0 \\
\hline July & 3 & 2 & 4 & 5 & 2 \\
\hline September & 7 & 9 & 6 & 9 & 3 \\
\hline October & 6 & 10 & 12 & 10 & 4 \\
\hline November & 7 & 11 & 13 & 9 & 3 \\
\hline December & 5 & 8 & 9 & 8 & 1 \\
\hline TOTAL & 48 & 54 & 60 & 56 & 18 \\
\hline
\end{tabular}

$\mathrm{P}>0.005$.

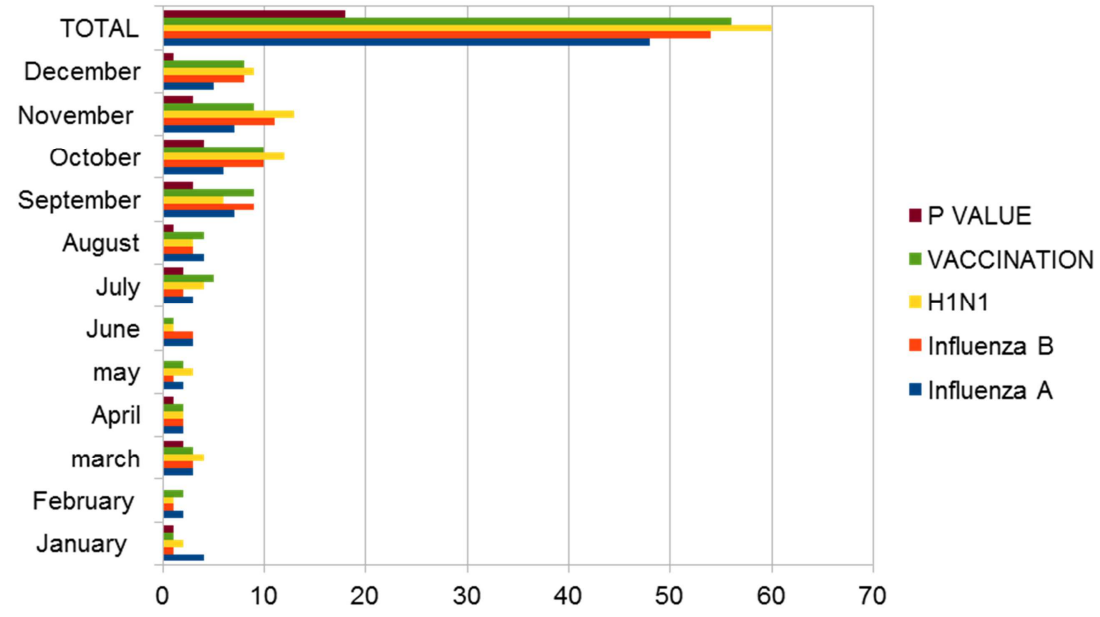

Figure 3. Effect of Influenza Vaccination of adult on Infection Rates.

Table 4. Risk factor with influenza RISK FACTOR.

\begin{tabular}{lllll}
\hline Month & NO & Influenza A & Influenza B & H1N1 \\
\hline Bronchial Asthma & 16 & 6 & 7 & 3 \\
Diabetics & 23 & 9 & 6 & 6 \\
Heart disease & 16 & 5 & 5 & 5 \\
Respiratory secretions & 19 & 5 & 5 & 4 \\
pneumonia & 12 & 3 & 4 & 4 \\
Immunocompromised & 9 & 3 & 3 & 2 \\
Pregnancy & 4 & 1 & 43 & 25 \\
TOTAL & 99 & 32 & & \\
\hline
\end{tabular}

\section{Discussion}

Influenza is an acute respiratory illness, caused by influenza A, B, and C viruses, that occurs in local outbreaks or seasonal epidemics. Clinical illness follows a short incubation period and presentation ranges from asymptomatic to fulminate, depending on the characteristics of both the virus and the individual host. Influenza A viruses can also cause sporadic infections or spread worldwide in a pandemic when novel strains emerge in the human population from an animal host. New approaches to influenza prevention and treatment for management of both seasonal influenza epidemics and pandemics are desirable [10].

The updated recommendations of the Advisory Committee on
Immunization Practices (ACIP) regarding the use of seasonal influenza vaccines is available at: Prevention and Control of Seasonal Influenza with Vaccines, 2018-2019. Routine annual influenza vaccination is recommended for all persons age 6 months and older who do not have contraindications. A licensed, recommended, and age-appropriate vaccine should be used. (http://www.nfid.org/influenza [11]. In our study Laboratoryconfirmed influenza was detected in 361of 1271 (28.4\%) in the (128 (10.07\%) had influenza A, and 144 (11.3\%) had influenza B by RT-PCR), and H1N1 in 89 (7-\%)-and one case influenza B, H1N1 by PCR.

The Centers for Disease Control and Prevention (CDC) recommends annual flu vaccination for everyone age 6 months or older. Each year's seasonal flu vaccine contains protection from the three or four influenza viruses that are 
expected to be the most common during that year's flu season [11].

The overall influenza vaccine protective effectiveness measured by RT-PCR to diagnose influenza among all study participants was $63 \%(95 \% \mathrm{CI}, P=.04)$ in the adjusted analysis (Tables 2, 3). Protective effectiveness for healthy children and adolescents was 59\% (95\% CI) and adult 63\% (95\% CI).

It is thought that all of these measures may reduce the risk of transmission of influenza though in fact the evidence base is weak. Expert opinion is supportive, There is some evidence supporting that surgical masks worn by ill people may reduce infections among close contacts when influenza is circulating. Avoiding close contact with sick people, e.g. by maintaining a distance of at least one meter from someone with symptoms of influenza, and avoiding crowded situations. When distance cannot be maintained, reducing the time of close contact with people who might be ill may be an option, Avoiding touching one's eyes, nose or mouth. Viruses may be spread when a person touches something that is contaminated with the virus and then touches his or her eyes, nose, or mouth [12].

Collection of appropriate respiratory samples and the application of a laboratory diagnostic test is required to establish a definitive diagnosis. Proper collection, storage and transport of respiratory specimens is the essential first step for laboratory detection of influenza virus infections. Laboratory confirmation of influenza virus from throat, nasal and nasopharyngeal secretions [13].

In our study influenza like illness, had risk factor29 cases which include, bronchial asthma found in 16 cases, diabetics in 23 , and heart disease in 16 cases, and pneumonia in 12 cases, and respiratory secretion in 19 cases, pregency in 4 cases, 12 cases were hospitalized for in respiratory infection pneumonia; there were 19 had cases had increase respiratory secretion and no deaths due to these infections, 16 due to heart disease and 9 due to cancer; 12 October 2018 - At the start of the flu season in the Northern hemisphere, WHO launched to an in-depth multimedia package on influenza, including the lessons we can learn from previous flu pandemics, how prepared we are for another one, and how work on seasonal flu can help countries to prepare for future pandemics.

When comparing 1272 swab from healthy children and adolescents and adult, 362 had influenza infection, 81 who received influenza vaccine in children, 56 who receive influenza vaccine in adult, there were no significant differences respectively in children who experienced influenza infection 199 vs adult 162, $P=.24$ ), However, more children and adolescents vaccinated with influenzavaccine reported.

The flu comes around every year, everywhere - and children are among some of the most vulnerable. We asked children all over the world to illustrate this spotlight on flu, which includes 5 Things To Do If You Have The Flu and 5 Ways to Avoid Getting The Flu [14].

Influenza is a highly contagious viral infection of the nose, throat, and lungs that occurs most often in the late fall, winter, and early spring. It is a serious infection that affects between $5-20 \%$ of the US population annually. During the 2017-2018 season, an estimated 950,000 individuals were hospitalized and nearly 80,000 deaths occurred in the US from flu and flu-related complications [14].

\section{Recommendation}

Routine annual influenza vaccination is recommended for all persons age 6 months and older.

\section{References}

[1] Hayward AC, Fragaszy EB, Bermingham A, et al. Comparative community burden and severity of seasonal and pandemic influenza: results of the Flu Watch cohort study. Lancet Respir Med. 2014; 2 (6): 445-54.

[2] Ducatez MF, Pelletier C, Meyer G.: Influenza D virus in cattle, France, 2011-2014. Emerg Infect Dis. 2015; 21 (2): 368-71.

[3] Cowling B, Chan K-H, Fang VJ, Cheun CYK, Fung ROP, Wai W. et al.: Facemasks and hand hygiene to prevent influenza transmission in households: a cluster randomized trial Annals of Internal medicine 2016; 151: 437-446.

[4] Kilbourne E.: Influenza pandemics of the 20th century. Emerg Infect Dis; 2006.

[5] Thompson WW, Shay DK, Weintraub E, et al.: Mortality associated with influenza and respiratory syncytial virus in the United States. JAMA. 2013; 289 (2): 179-86.

[6] Webster RG, Monto AS, Braciale TJ, Lamb RA (editors). Textbook of Influenza. 2nd ed. Chichester: John Wiley \& Sons Ltd; 2013.

[7] Nair H, Abdullah Brooks W, Katz M et al. Global burden of respiratory infections due to seasonal influenza in young children: a systematic review and meta-analysis. lancet 2011; 378: 1917-3.

[8] WHO: recommended surveillance standards, 2017.

[9] Center for Disease Control and Prevention (CDC (Influenza (Flu) - Center for Disease Control and Prevention (CDC), USA, March 1, 2018).

[10] www.health.qld.gov.au/clinical-practice/guidelinesprocedures/diseases-infection/immunisation/research, 2018.

[11] http://www.nfid.org/influenza, 2019.

[12] Chartrand C, Leeflang MM, Minion J, Brewer T, Pai M.: Accuracy of rapid influenza diagnostic tests: a meta-analysis. Ann Intern Med. 2012 Apr 3; 156 (7): 500-11.

[13] Merckx J, Wali R, Schiller I, Caya C, Gore GC, Chartrand C et al.: Diagnostic Accuracy of Novel and Traditional Rapid Tests for Influenza Infection Compared With Reverse Transcriptase Polymerase Chain Reaction: A Systematic Review and Meta-analysis. Ann Intern Med. 2017 Sep 19; 167 (6): 394.

[14] lancet, http://doii.orgl10.1016/so140-6736, 2018. 